\title{
Unveiling the nanoscale heterogeneity controlled deformation of thermosets
}

\author{
Jérémy Chevalier ${ }^{\mathrm{a}, 1, *}$, Laurence Brassart ${ }^{\mathrm{d}}$, Frédéric Lani $^{\mathrm{b}}$, Christian Bailly ${ }^{\mathrm{c}}$, Thomas Pardoen ${ }^{\mathrm{a}, 1}$, Xavier P. Morelle $^{\mathrm{a}, 1, *}$ \\ ${ }^{a}$ Institute of Mechanics, Materials ad Civil Engineering (iMMC), Université catholique de Louvain, 1348, Louvain-la-Neuve, Belgium \\ ${ }^{b}$ Any-Shape s.a., 4400, Flémalle, Belgium \\ ${ }^{c}$ Institute of Condensed Matter and Nanosciences (IMCN), Université catholique de Louvain, 1348, Louvain-la-Neuve, Belgium \\ ${ }^{d}$ Department of Materials Science and Engineering, Monash University, Clayton, VIC 3800, Australia
}

\begin{abstract}
The deformation behavior of polymer materials below their glass transition temperature involves a complex intermingling of elasticity, of viscoplastic yielding with softening and large strain hardening, and of significant rate, temperature and pressure dependency. During unloading, the response exhibits large non-linearity as well, combining forward and backward creep. Even though the nature of the atomistic underlying mechanisms is relatively well understood, a full picture of the interactions between the elementary distortions mechanisms and their collective organization capturing the complexity of the macroscopic behavior is still lacking. Here, we propose a mesoscale approach based on the heterogeneous activation of molecular level shear transformation zones and we show that it is rich enough to unravel the physical origin of most aspects of the macroscopic viscoplastic response. The study is based on a wide microand macro-mechanical test campaign on a thermoset material. In addition to providing a full characterization of all macroscopic properties, evidence of a complex rate reversal mechanism upon unloading is given. Other experiments provide insights about the characteristic length scales at play. Compared to existing continuum models involving typically more than 25 parameters, the present formalism is based on only 7 physical parameters while allowing quantitative predictions of all the experimental data. The present approach opens new avenues for the prediction of the mechanical response of a variety of polymer applications in confined state such as in composites or in adhesive joints. It also offers a new line of thought to address the mechanics of polymers at small and intermediate length scales.
\end{abstract}

Keywords: deformation micro-mechanisms, discrete and heterogeneous plasticity, mesoscale modeling, STZ dynamics, glassy polymers

\section{Introduction}

Amorphous polymers are one of the most ubiquitous materials in our current daily lives, ranging from simple kitchenware made of polystyrene to advanced epoxy-based composite panels used in aircrafts and cars. The low density, low cost, easy processability, and durability of glassy polymers make them versatile in terms of applications and attractive for structural solutions undergoing moderate loading levels. This attractive set of properties comes from their macromolecular nature (Meijer and Govaert, 2005), that distinguishes them from hard structural materials like polycrystalline metallic alloys. Their mechanical response in tension and compression shows a peculiar behavior, exhibiting post-yield strain softening, large strain hardening with significant strain rate, temperature and pressure dependence, and strong non-linearity upon unloading (e.g. Boyce et al. (1988); Fiedler et al. (2001); Gerlach et al. (2008); Poulain et al. (2014); Morelle et al. (2017)). Over the years, a number of (visco)elastic-viscoplastic constitutive models have been developed in an attempt to capture the full spectrum of post-yield deformation phenomena of glassy polymers. Most of these models originate from the pioneer 1D elastic-viscoplastic model of Haward and Thackray (1968) where the glassy polymer response is decomposed into (i) an intermolecular resistance contribution corresponding to the thermally-activated plastic flow, and (ii) a configurational entropy resistance contribution,

${ }^{*}$ Corresponding author 
borrowed from rubber elasticity, to account for the large strains rehardening process due to finite chain extension and alignement. If these models were successively refined and extended (e.g. Boyce et al. (1988); Wu and Van Der Giessen (1993); Tervoort et al. (1996); Bergstrom and Boyce (2001); Anand and Gurtin (2003); Anand et al. (2009); Ames et al. (2009); Srivastava et al. (2010); Nguyen et al. (2016); Gudimetla and Doghri (2017)), they still typically require a large number of phenomenological parameters (at least 20, and often many more) with limited physical basis. For instance, even with recent models, a heuristic law is usually used to describe the post-yield softening (e.g. Poulain et al. (2013)), while the superposition of several kinematic hardening components is required to capture the experimentally-observed strong non-linearity effects upon unloading (Anand et al., 2009; Senden et al., 2010). Moreover, even the most elaborate models do not rely on purely micro-mechanical ground to explain the origin of the complex macroscopic behavior in the glassy state. Indeed, while a globally-accepted molecular deformation picture is available above $T_{g}$, known as the tube model (Wang et al., 2013), the physical description of the deformation micro-mechanisms in the amorphous state (below $T_{g}$ ) is still open to debate (Budrikis et al., 2017). The present work aims at providing a possible generic answer for the glassy regime behavior through a bottom-up mesoscale modeling approach, validated for the case of the RTM6 epoxy resin.

Due to the lack of experimental observations available at the microscopic scale for amorphous polymers, the only clues on the possible molecular resistance mechanisms involved in the glassy state were either : (i) suggested through molecular yield theories developed in the late sixties by Ree and Eyring (1955), Robertson (1966), Bowden and Raha (1974) ,Argon (1973), each assuming a different molecular rearrangement or conformational change (such as double kinks, dihedral flips and twists, and disclination loops) as the possible carrier of local plasticity ; or (ii) unravelled via molecular dynamics (MD) simulations (e.g. Hoy and Robbins (2008); Rottler (2009); Chen and Schweizer (2011); Jatin et al. (2013); Sundararaghavan and Kumar (2013)) confirming that several of the envisioned molecular rearrangements can indeed occur during plastic flow. MD simulations also revealed the role played by nanoscale heterogeneities, and their dynamic evolution, in the microstructural state (Falk and Langer, 1998; Ediger, 2000). However, MD-based analysis is typically restricted to very short time scales and to small material volumes, which limits its practical use with respect to real-life experimental conditions or to the longer time scales associated with thermally-activated mechanisms. Moreover, while both approaches - molecular yield theories and MD simulations - helped clarifying the atomistic elementary mechanisms of plasticity in glassy polymers, a clear picture of how the interactions between these elementary events and their collective organization affect and explain the macroscopic response of amorphous polymers is still lacking.

Regardless of the exact nature of the molecular rearrangements, it is now admitted that the flow behavior in glassy polymers originates from the nucleation and cooperative interaction of localized conformational changes in the molecular chains (Argon, 2013; Malekmotiei et al., 2015). In particular, Oleynik (1989) experimentally observed through differential scanning calorimetry of deformed glassy polymers that a major part of the energy stored during plastic deformation cannot be attributed to conformational changes alone, but rather to the elastic interaction of these localized plastic events with the surrounding matrix. Based on these pioneering observations, Hasan and Boyce (1995) were the first to demonstrate that a model based on the thermally-activated nature and highly heterogeneous microstructural state of glassy polymer can successfully predict their full viscoplastic behavior. Hence, discrete irreversible rearrangements, encompassing any kind of glide, slip or shear-rotation of the polymer chains, along with their interaction with the microstructural surrounding, can be considered as the elementary micro-mechanisms driving plasticity in glassy polymers (Stachurski, 1997; Argon, 2013).

In the larger field of plasticity of amorphous materials, the concept of discrete plasticity events interacting through a polarized stress field is not new (see e.g. Argon (1973); Argon and Bessonov (1977); Argon (2013); Bowden and Raha (1974); G'Sell (1986); Mangion et al. (1992)). In particular, Argon (1979) proposed a framework, originally developed for metallic glasses, based on atomic clusters of conformational changes, called "Shear Transformation Zones" (STZs). The shear transformation process is governed by an activation energy quantified by the increment of elastic energy stored in a STZ and its immediate surrounding when it experiences a local transformation shear strain. The pioneering work by Argon (1979) has been widely used as a basis for analyzing the dynamics of deformation in the field of metallic glasses. For instance, implementations of extensions of the initial STZ framework were successful in modelling both the viscoplasticity and the impact of the thermo-mechanical history in metallic glasses (e.g. Falk and Langer (1998); Falk et al. (2004); Shi and Falk (2005); Langer (2008)), or to capture the complex shear pattern observed experimentally (Budrikis et al., 2017). Nonetheless, the concepts of local rearrangements through shortrange order in an atomic environment are also relevant in a macromolecular context (Argon, 2013; Wang et al., 2014). 
Therefore, the STZ concept has recently been the subject of increasing attention in the field of polymer glasses as well. Following the early extension to polymers by Argon and co-workers (Mott et al., 1993; Bulatov and Argon, 1994; Argon et al., 1995), more recent studies have used implementations similar to Argon's STZ model in an attempt to measure the STZ size (Samadi-Dooki et al., 2016), to model the viscoplastic behavior in compression (Voyiadjis and Samadi-Dooki, 2016), to capture the size effect observed in nanoindentation (Voyiadjis et al., 2018) or to determine the impact of confinement on the rheological properties (Masurel et al., 2015, 2017).

In this work, inspired by the implementation elaborated by Schuh and coworkers (Homer and Schuh, 2009; Li et al., 2013; Harris et al., 2016) of Argon's STZ model for metallic glasses, we have developed a mesoscale model for glassy polymers based on random, heterogeneous activation of STZs. In this modelling framework, STZs first tend to initiate randomly in the material, around regions with higher mobility or excess energy, and hence lower activation energy barrier. In a second stage, STZs start interacting with each other, through the elastic field generated in their surroundings after activation. Due to the polarized mechanical field, STZs tend to progressively activate in the form of larger clusters by joining with just-activated neighboring STZs, eventually leading to local micro shear band propagation, building strong backstress in the material. Thanks to its conceptual simplicity, the model requires the identification of only seven parameters, out of which five are not directly obtained from experimental data. The model is calibrated and validated based on a large experimental dataset obtained on the RTM6 epoxy resin, a highly cross-linked epoxy resin widely used in aeronautic and space applications as a matrix for fiber reinforced composites. Experimental results include the compression response under varying strain rates and temperatures, including loading and unloading, as well as creep tests at different levels of applied load (Morelle, 2015; Morelle et al., 2017).

We show here that the proposed formalism is rich enough to predict the full stress-strain response and to quantitatively reproduce all the complex deformation phenomena associated with glassy polymers mechanical response, including post-yield strain softening and non-linear unloading behavior, without relying on any viscoelastic or plastic ad hoc continuum assumptions. One key feature of our mesoscale model is the generation of large backstress as a result of the interactions between randomly generated STZs. This allows, among other things, the prediction of successive backward and forward creep upon partial unloading, also called rate-reversal phenomenon. The small number of ingredients used to predict a large range of phenomena supports the relevance of the physically proposed micromechanism, opening many avenues to address intermediate scale mechanical phenomena and bridging atomistic and continuum models.

The outline of this paper is the following. The model is presented first, including the formulation of the energy barrier, the addings specific to the present application on thermosets, the FE implementation and the identification of the model parameters. Section 3 combines the results in terms of validation and parameters study with the discussion about the mechanisms unravelled by the STZ based simulations. Section 4 provides additional experimental data confirming the predicted heterogeneous deformation pattern.

\section{Modeling framework}

A STZ is a small volume of material which can undergo a thermally-activated, stress-induced change of configuration involving a shear transformation strain $\Delta \varepsilon_{i j}^{*}$ (Fig. 1c). The underlying molecular mechanism is left unspecified, and can be a dihedral flip, a crankshaft, a chain slip, etc. The transformation is stable, leading to a permanent deformation and can only be reversed if an energy barrier is overcome again, which is unlikely by pure thermal activation. In two dimensions, we assume that the transformation strain is purely deviatoric, with maximum engineering shear strain $\gamma_{0}$ and shear angle $\omega$, respectively (Fig. 1c). In the following, we will refer to $\omega$ as the direction of activation, for simplicity.

The energy barrier for the transformation of a STZ is a material parameter that varies with local temperature, mechanical and microstructural state. Following Argon (1979), the amplitude $\Delta F_{0}$ of the energy barrier can be approximated by the elastic energy change associated with the shear transformation of a single spherical inclusion in an infinite elastic matrix, using Eshelby's solution (Eshelby, 1957). The expression of $\Delta F_{0}$ includes three contributions: i) $\Delta F_{\text {shear }}$ corresponds to the matrix strain energy associated with the shear deformation of the STZ, ii) $\Delta F_{\text {dil }}$ corresponds to the matrix strain energy associated with the effective temporary dilation $\varepsilon_{v \text {,eff }}$ necessary to activate a STZ, and iii) $\Delta F_{\text {coh }}$ is a cohesion term associated with the shearing of an unconstrained STZ, with $\hat{\tau}$ the intrinsic 


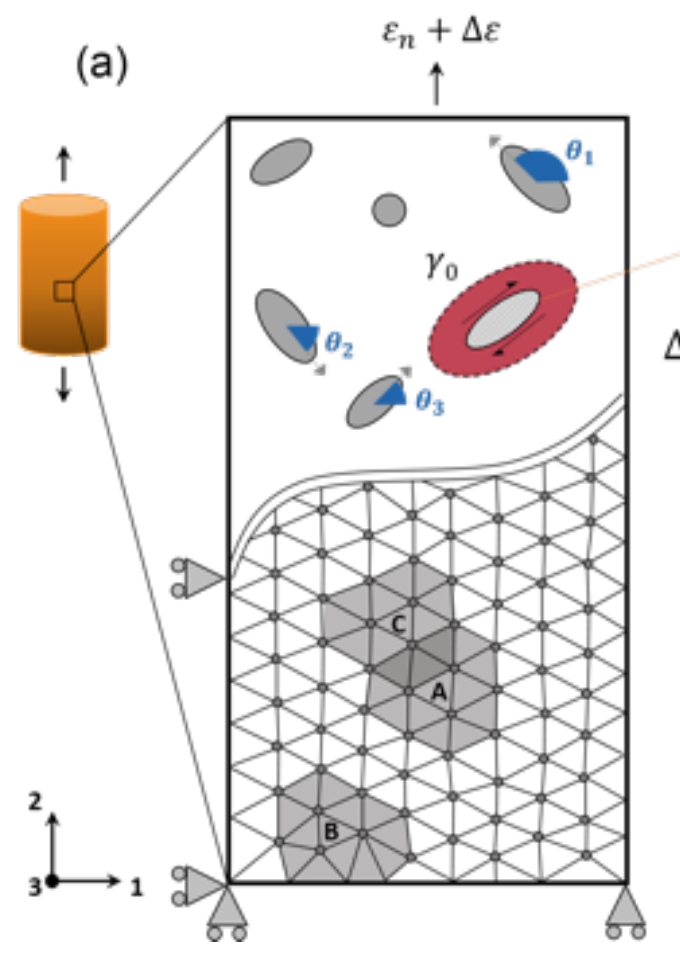

(b)

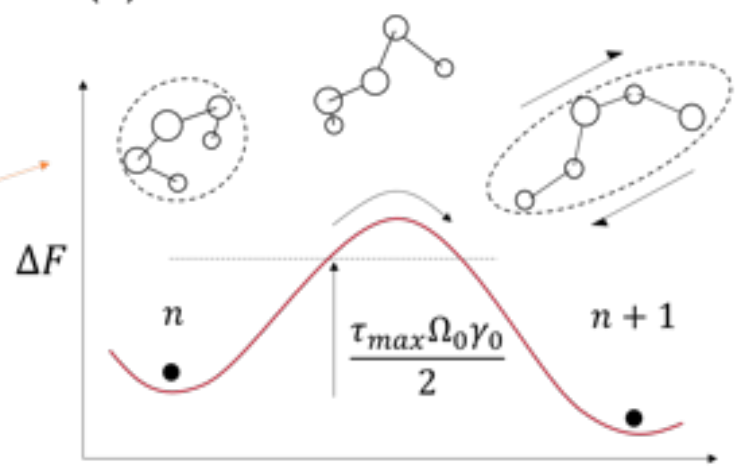

(c) $\left\langle\sigma_{i j}\right\rangle_{\mathrm{STZ}}=C_{i j k l}\left(\left\langle\varepsilon_{k l}\right\rangle_{\mathrm{STZ}}-\varepsilon_{k l}^{*}\right)$

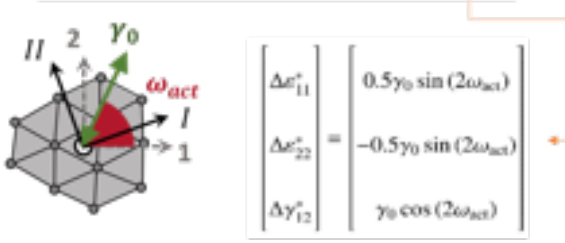

Figure 1: Schematic representation of the Shear Transformation Zone (STZ) modeling framework. A STZ is defined as a molecular cluster of polymer chains able to undergo a thermally-activated, stress-induced change of conformation. In the present FE framework, it is modelled as a central element and all the neighboring elements sharing a common node with it, as illustrated by the STZs A, B and C in grey. (a) A 2D plane-strain FE triangular mesh coupled to a KMC algorithm is used to describe the STZs interactions, mediated by their neighboring elastic field, and occuring at the mesoscale in a glassy polymer. (b) Upon applied external loading, at each time increment, each STZ is assessed to check if it has enough thermo-mechanical energy for a forward activation leading to (c) a deviatoric deformation of $\gamma_{0}$ in the local direction $\omega$ of maximum shear stress. After several activations, each STZ has an accumulated strain orientation defined by $\theta$. 
inter-molecular shear resistance. This energy barrier writes:

$$
\begin{aligned}
& \Delta F_{0}\left(T, \sigma_{n}\right)=\Delta F_{\text {shear }}+\Delta F_{\text {dil }}+\Delta F_{\text {coh }}=\frac{7-5 v}{30(1-v)} \mu(T) \gamma_{0}^{2} \Omega_{0}+\frac{2(1+v)}{9(1-v)} \mu(T) \varepsilon_{v, \text { eff }}^{2} \Omega_{0}+\frac{1}{2} \hat{\tau} \gamma_{0} \Omega_{0} \\
& \text { with } \varepsilon_{v, \text { eff }}=\varepsilon_{v}\left(1-\beta \frac{\sigma_{n}}{E(T)}\right) .
\end{aligned}
$$

In Eq. [1], $T$ is the absolute temperature, $v$ is the Poisson ratio, $\mu(T), \Omega_{0}(T)$ and $E(T)$ are respectively the temperaturedependent shear modulus, activation volume and Young's modulus, and $\varepsilon_{v}$ is the temporary activation dilation in the absence of stress. Compared to Argon's original expression, the effective temporary dilation $\varepsilon_{v \text {,eff }}$ has been introduced in order to account for the pressure dependence of the transformation. The effect of a pre-existing volumetric strain on the energy barrier is accounted for through the term $\beta \frac{\sigma_{n}}{E(T)}$, with $\sigma_{n}$ the stress normal to the transformation shear strain direction (see Fig. 1c), and $\beta$ a positive fitting parameter. Hence, $\sigma_{n} / E(T)$ either reduces or increases the effective, temporary volumetric strain required for transformation, depending on the sign of $\sigma_{n}$. Note that the effective temporary dilation does not depend explicitly on the hydrostatic stress, but only on the normal stress. Our results indicate that this additional dependency can describe the asymmetry between the tension and compression response, in agreement with experimental observations. This is demonstrated by Fig. 2e and $2 \mathrm{f}$ in SI. The temporary dilation is a transient state, only accounted for in the calculation of the energy barrier $\Delta F_{0}$ and it does not translate into an actual volumetric strain in the model.

In addition to the elastic energy associated with the transformation, the current microstructural state (i.e. local molecular mobility) and the chain stretching inside a given STZ are considered to have a direct influence on the amplitude of the energy barrier. In order to describe these effects, the following expression for an effective energy barrier, $\Delta F_{\text {eff }}$, was adopted, generalizing Eq. [1] :

$$
\Delta F_{\text {eff }}=\Delta F_{\text {shear }}+\left(\Delta F_{\text {dil }}+\Delta F_{\text {coh }}\right) g\left(f_{v}\right)+H(\lambda) \cos (2(\omega-\theta)),
$$

where $f_{v}$, called the local "free volume" content, is a phenomenological parameter characterizing the local excess energy or chain mobility, $\lambda$ is a measure of the polymer chain elongation, and the angles $\omega$ and $\theta$ respectively give the angle of the maximum shear stress $\tau_{\max }$ within the STZ domain and the averaged orientation of its accumulated transformation shear strain (see Fig. 1a and 1c). The functions $g\left(f_{v}\right)$ and $H(\lambda)$ will be described in more details later.

The expression for the rate of STZ activation $\dot{s}$ is borrowed from the transition state theory developed by Ree and Eyring (1955) and follows Argon (1979):

$$
\dot{s}=v_{0} \exp \left(-\frac{\Delta F_{\mathrm{eff}}-\tau_{\max } \Omega_{0} \gamma_{0} / 2}{k_{B} T}\right)
$$

where $v_{0}$ is the attempt frequency, taken as equal to the Debye frequency and $k_{B}$ is the Boltzmann constant. The term $\tau_{\text {max }} \Omega_{0} \gamma_{0} / 2$ is the mechanical energy supply that drives the transformation.

The kinetics of STZ activation is governed by a time-dependent Kinetic Monte Carlo (KMC) algorithm (Dawnkaski et al., 1995). The algorithm determines whether a STZ is activated during a small time increment $\Delta t$, based on the following probability of activation $P_{\text {act }}$ :

$$
P_{a c t}=\dot{s} \Delta t
$$

The probability of activation of the STZ is then compared to a randomly generated number $\xi_{1} \in[0,1]$. If $\xi_{1}<P_{\text {act }}$, the STZ is activated, and the following transformation strain increment is applied to the STZ:

$$
\left[\begin{array}{l}
\Delta \varepsilon_{11}^{*} \\
\Delta \varepsilon_{22}^{*} \\
\Delta \gamma_{12}^{*}
\end{array}\right]=\left[\begin{array}{c}
0.5 \gamma_{0} \sin \left(2 \omega_{\text {act }}\right) \\
-0.5 \gamma_{0} \sin \left(2 \omega_{\text {act }}\right) \\
\gamma_{0} \cos \left(2 \omega_{\text {act }}\right)
\end{array}\right] .
$$


The angle of activation $\omega_{\text {act }}$ is assumed identical to $\omega$, the direction of $\tau_{\max }{ }^{1}$.

\subsection{Effect of free volume}

As said above, in the context of this work, the free volume is a phenomenological parameter characterizing the local molecular mobility at the scale of the STZ regions and of their close neighborhood. The analogy between the local free space and a local variable facilitating further activations is easy to visualize. However, while the concept of free volume has been largely reviewed in the literature of glassy polymers, there is no general consensus on the link between regions of higher free space and regions prone to shear deformation, neither that there is a consensus regarding the fact that local conformational changes lead to a local increase of free space. Hence, in this work, the concept of free volume is accounted for in a very general way and a high free volume content is associated to an energy landscape locally favorable for a STZ activation. It represents the local excess of energy and steric compliance available for the STZ activation that is not included into the local continuum elastic stress field accounted for by the finite element description (to be described later).

In our model, the free volume content is described by a normalized state variable $f_{v}$ associated with each STZ and varying between 0 and 1 . As discussed by Argon, the local temporary dilation around an STZ during its transformation involves a local increase of the amount of free-volume, i.e. of chain mobility (Argon, 1973; Hasan et al., 1993). Each activation thus leads to an increase of the local free volume content by an amount $\Delta f_{v}^{+}$, taken of the form:

$$
\Delta f_{v}^{+}=\varepsilon_{v, \mathrm{eff}}\left(1-f_{v}\right)
$$

where $\varepsilon_{v, \text { eff }}$ is the effective temporary dilation, previously defined in Eq. [1].

The local free volume content facilitates subsequent activation of the STZ by reducing the dilational strain energy required to allow the transformation, as well as the associated cohesion term. In Eq. [2], the role of excess free volume is accounted for through the function $g\left(f_{v}\right)$ given by Argon in Argon (1979); Li et al. (2013) :

$$
g\left(f_{v}\right)=\frac{1+0.8\left[\varepsilon_{v, \mathrm{eff}}\left(1-f_{v}\right)\right]^{2}}{1+0.8 \varepsilon_{v, \mathrm{eff}}^{2}} .
$$

Alongside free volume generation, free volume annihilation occurs due to diffusive rearrangements, a process also referred to as physical aging. Diffusive rearrangement is also a thermally-activated process, with a rate of activation of the form (Argon, 1979; Li et al., 2013):

$$
\dot{s}_{D}=\left(1-f_{v}\right) v_{D} \exp \left(-\frac{\Delta F_{D}\left(1-f_{v}\right)}{k_{B} T}\right),
$$

where $\Delta F_{D}$ is the energy barrier for diffusive rearrangement and $v_{D}$ is a frequency factor, similar to $v_{0}$ in Eq. [1]. $\Delta F_{D}$ is considered equal to $\Delta F_{0}$. In the $\mathrm{KMC}$ algorithm, a probability of diffusive rearrangement in each $\mathrm{STZ}$ is calculated as $P_{D}=\dot{s}_{D} \Delta t$, and rearrangement occurs if $\xi_{2}<P_{D}$, where $\xi_{2} \in[0,1]$ is a second randomly generated number. Once activated, diffusive rearrangement leads to a decrease $\Delta f_{v}^{-}$of the local state variable $f_{v}$ according to Argon (1979):

$$
\Delta f_{v}^{-}=\varepsilon_{v, \text { eff }} f_{v}
$$

\subsection{Effect of finite chain network extensibility}

At large strain, like most glassy polymers, the highly cross-linked epoxy resins exhibit a strong strain hardening behavior, through chain reorientation and cross-links/entanglements locking mechanisms. Strain hardening can thus be understood as a decrease of the number of possible conformations of the network when the chains align in the main loading direction, either because all possible rearrangements (chain rotation, dihedral flips,...) in the loading direction have been exhausted, or because of increasing geometric constraint hindering molecular reconfigurations as

\footnotetext{
${ }^{1}$ This is different from the model of Schuh and Homer (Homer and Schuh, 2009; Li et al., 2013; Harris et al., 2016), where the orientation angle of the STZ varies stochastically around the direction of $\tau_{\max }$ to account for the increasing variability in activation orientation when the temperature is high and/or the stress is low.
} 
the chains become closer (Jatin et al., 2013). In the proposed model, the probability of activating a STZ in a particular direction should decrease as the number of prior activations in that direction increases. This effect is accounted for through the contribution $H(\lambda)$ in the effective energy barrier (Eq. [2]), where $\lambda$ quantifies the degree of elongation of a chain. While previous studies have demonstrated that strain hardening in glassy polymer could not be considered as fully entropic in nature (Van Melick et al., 2003; Hoy and Robbins, 2006, 2007, 2008), a neo-Hookean model can still adequately quantify the elastic energy associated with chain elongation:

$$
H\left(\lambda_{1}, \lambda_{2}, \lambda_{3}\right)=\Psi\left(\lambda_{1}^{2}+\lambda_{2}^{2}+\lambda_{3}^{2}-3\right)
$$

where $\lambda_{i}(i=1,2,3)$ are the principal stretches in the STZ and $\Psi$ is a constant having units of energy. In the original Neo-Hookean model, the constant $\Psi$ is related to the number $N_{e}$ of entanglements or cross-links, $\Psi=\frac{1}{2} N_{e} k_{B} T$. Here however, since the strain hardening in RTM6 has been shown to be temperature independent (Morelle et al., 2017), $\Psi$ has been fitted to capture the amplitude of strain hardening at room temperature and is kept constant.

The penalty associated with successive activation of a STZ in the same direction should not apply when the direction $\omega$ of $\tau_{\max }$ and the averaged shear strain direction $\theta$ of the accumulated past transformations differ, or, at least, not to the same extent. To account for this directional hardening effect, the function $H(\lambda)$ is multiplied by the factor $\cos (2(\omega-\theta))$ in Eq. [2], therefore accounting for the prior state of stretch (i.e. amplitude and orientation) of a given STZ.

\subsection{Finite Element implementation}

The two-dimensional (2D) model is implemented within the commercial finite element package (Abaqus, 2012) using several user subroutines (SDVINI, URDFIL), a user-defined material subroutine UMAT and a user-defined subroutine for the KMC algorithm (see Fig. S1 in supplementary for detailed subroutines call sequence and associated flow-chart). Each STZ comprises several elements of the 2D triangular mesh. It consists in a central element and all its neighboring elements sharing at least one node (Fig. 1c). Each element is considered as the center of a STZ, which means that different STZs can share common elements, accounting for the fact that a given chain segment can be involved in several possible STZs. This modeling approach thus explicitely accounts for the short-range interactions between STZs. The STZs are composed of an average of 13 elements, which, as demonstrated by Homer and Schuh (2009), is large enough to prevent significant discrepancy when compared to Eshelby single inclusion solution, while being a reasonable compromise in terms of computational time.

At any increment of the simulation, a STZ can undergo a shear transformation increment $\Delta \varepsilon_{i j}^{*}$ given by Eq. [5] applied to all the elements in the STZ, following the KMC algorithm previously described. Therefore, during a given time increment, several STZs can activate simultaneously, while each element can be activated several times as elements belong to several STZs. The local and average stress in any STZ evolves with the following constitutive relationships:

$$
\sigma_{i j}=C_{i j k l}\left(\varepsilon_{k l}-\varepsilon_{k l}^{*}\right), \quad\left\langle\sigma_{i j}\right\rangle_{\mathrm{STZ}}=C_{i j k l}\left(\left\langle\varepsilon_{k l}\right\rangle_{\mathrm{STZ}}-\varepsilon_{k l}^{*}\right),
$$

where $C_{i j k l}$ is the isotropic elastic stiffness tensor and $\varepsilon_{k l}^{*}$ the accumulated transformation strain applied to the STZ. This transformation strain is thus zero as long as the STZ has not been activated. The notation $\langle\cdot\rangle_{\text {STZ }}$ refers to the volume average over the elements of a STZ. The transformation strain increment associated with a STZ activation is accommodated elastically by the surrounding material, in such a way that mechanical equilibrium is satisfied (in the finite element sense). The resulting heterogeneous stress field impacts subsequent activation events through $\tau_{\max }$ in Eq. [3], estimated in each STZ as the average maximum shear stress, $\left\langle\tau_{\max }\right\rangle_{\mathrm{STZ}}$. Once again, it is important to realize that the FE model is not used to estimate the mechanical contribution associated to the energy barrier which is assumed to be adequately predicted with Eq. [1]. The FE model is only used to calculate the stress distribution related to the shear transformation strain.

The simulations are carried out on a 2D mesh containing over 100,000 second-order plain strain elements (CPE6), which ensures the model to be a scale above the size of a fully propagated shear band. A mesh convergence study was made to ensure the representativeness of the model response. Plane strain tension or compression is applied in direction 2, corresponding to a prescribed macroscopic strain rate $\dot{\bar{\varepsilon}}_{22}$. A fully-reversed loading is obtained by first 
Table 1: List of the model parameters. The symbols $\dagger$ denote the fitting parameters while the rest are experimentally determined.

\begin{tabular}{l|c|c|c|c|c|c|c|c|c|c|c}
\hline Parameter & $\begin{array}{c}\Omega_{0} \\
{\left[\mathrm{~nm}^{3}\right]}\end{array}$ & $\begin{array}{c}d \Omega_{0} / d T \\
{\left[\mathrm{~nm}^{3} / K\right]}\end{array}$ & $\begin{array}{c}\gamma_{0}^{\dagger} \\
{[-]}\end{array}$ & $\begin{array}{c}\varepsilon_{v}^{\dagger} \\
{[-]}\end{array}$ & $\begin{array}{c}\beta^{\dagger} \\
{[-]}\end{array}$ & $\begin{array}{c}\Psi^{\dagger} \\
{[\mathrm{eV}]}\end{array}$ & $\begin{array}{c}\hat{\tau}^{\dagger} \\
{[\mathrm{MPa}]}\end{array}$ & $\begin{array}{c}E(296 \mathrm{~K}) \\
{[\mathrm{GPa}]}\end{array}$ & $\begin{array}{c}E(373 \mathrm{~K}) \\
{[\mathrm{GPa}]}\end{array}$ & $\begin{array}{c}E(423 \mathrm{~K}) \\
{[\mathrm{GPa}]}\end{array}$ & $\begin{array}{c}v \\
{[-]}\end{array}$ \\
\hline Value & 2.5 & 0.0148 & 0.2 & 0.27 & 0.5 & 0.5108 & 120 & 3.0 & 2.25 & 2.15 & 0.34 \\
\hline
\end{tabular}

applying compression up to $\bar{\varepsilon}_{22}=-0.6$ followed by tension up to $\bar{\varepsilon}_{22}=0.55$. The creep simulations consist in loading the model in compression up to $\bar{\varepsilon}_{22}=-0.35$ and then partially unloading it down to a prescribed value of the reaction force maintained during 50s. The four edges of the rectangular model are kept straight to enforce periodicity. The effective (homogenized) stress-strain response $\left(\bar{\sigma}_{i j}, \bar{\varepsilon}_{i j}\right)$ of the polymer is given by the volume average of the local stress fields:

$$
\bar{\varepsilon}_{i j}=\left\langle\varepsilon_{i j}\right\rangle, \quad \bar{\sigma}_{i j}=\left\langle\sigma_{i j}\right\rangle
$$

where the symbol $\langle\cdot\rangle$ indicates a volume average over the total simulation volume.

\subsection{Identification of model parameters}

The parameters of the model are listed in Table 1. Besides the usual mechanical properties such as $E$ and $v$, several parameters can be directly extracted from experimental results. Indeed, the activation volume can be calculated from compression tests at different strain rates using Eyring equation for the thermally-activated dependence of the plastic flow of a glassy polymer (Eyring, 1936):

$$
\ln \dot{\varepsilon}=\ln (K)-\frac{\Delta F_{0}}{k_{B} T}+\frac{\sigma \Delta v^{*}}{k_{B} T}
$$

where $\dot{\varepsilon}$ is the strain rate, $K$ is a rate constant, $\Delta F_{0}$ is an energy barrier, $\sigma$ is the stress and $\Delta v^{*}=\Omega_{0} \gamma_{0}$ is the apparent activation volume. The evaluation of $\Delta v^{*}$ is described in more details in Morelle (2015). Experimental tests on RTM6 for a variety of loading conditions including jumps of strain rate, stress relaxation and strain recovery consistently gave an average value of $\Delta v^{*}=0.5 \mathrm{~nm}^{3}$ at room temperature (296K) (Morelle, 2015). On the other hand, the rate of change in activation volume with temperature, $d \Omega_{0} / d T$, was determined from Eq. [13] based on experimental data for pure compression tests at $296 \mathrm{~K}, 373 \mathrm{~K}$ and $423 \mathrm{~K}$.

A direct measurement of $\gamma_{0}$ is not possible and only molecular dynamics simulations can provide a direct estimate. Bulatov and Argon (1994) estimated the amplitude of $\gamma_{0}$ to be around 0.15 for polypropylene. This value is believed to slightly vary from one glassy polymer system to another (Argon, 2013). Here, the value $\gamma_{0}=0.2$ was selected to reproduce the experimental compression curve at room temperature, which implies that $\Omega_{0}=\Delta v^{*} / \gamma_{0}=2.5 \mathrm{~nm}^{3}$. By definition, the activation volume is related to the size of an STZ as the elementary deformation volume, and the full model size can therefore be estimated to be around $100 \mathrm{~nm} \times 200 \mathrm{~nm}$.

Finally, the parameters $\Psi, \beta, \hat{\tau}$ and $\varepsilon_{v}$ were selected to adequately fit the amplitude of the hardening and of the softening parts of the stress-strain response of RTM6 under compression (properly correcting for the fact that the simulations are in 2D plane strain and the tests in pure compression). The relative influence of the key parameters value $\left(\gamma_{0}, \varepsilon_{v}, \beta, \hat{\tau}\right.$, and $\left.\Psi\right)$ on the effective stress-strain response of the computational model can be found in Fig. S2 in SI, where it is compared to a representative experimental compression curve at room temperature at a true strain rate of $0.01 \mathrm{~s}^{-1}$ from which they were identified.

\section{Results and discussion}

In order to validate the model with the set of parameters identified above, the predictions of the FE simulations were compared to different experimental results with increasing degree of complexity: (i) the large strain post-yield response under compressive (monotonic) loading at different temperatures; (ii) the non-linear unloading behavior and Bauschinger effect associated with fully-reversed loading; and (iii) the forward creep, backward creep, and intermediate rate-reversal phenomena observed when the applied force is held constant after different levels of unloading. 
In each case, the micro-mechanical origin of the observed macroscopic phenomena is discussed based on the model mesoscopic description of the STZs dynamics.

\subsection{Large strain response under compression}

The effect of temperature on the uniaxial compression response is illustrated in Fig. $2 \mathrm{a}$ for $T=296 \mathrm{~K}, 373 \mathrm{~K}$ and $423 \mathrm{~K}$, and for an applied strain rate of $0.01 \mathrm{~s}^{-1}$. The curve corresponding to $T=296 \mathrm{~K}$ was used to identify the model parameters, while the other two curves are model predictions. The numerical results show that the temperature dependence of RTM6 mechanical response is properly predicted based on the experimentally-identified temperature dependence of the activation volume and mechanical properties (i.e. $E, \mu$ ). While the softening and hardening parts of the curves are perfectly captured by the model, the pre-yield non-linearity and the yield stress are respectively underand overestimated. These discrepancies, which tend to vanish at higher temperature, will be discussed in more details in a later section.

Fig. $2 \mathrm{~b}$ also shows the evolution of the homogenized free volume state variable $\left\langle f_{v}\right\rangle$ during a compression at $T=296 \mathrm{~K}$ at a strain rate of $0.01 \mathrm{~s}^{-1}$. The free volume progressively increases in the first stages of the simulation, before abruptly rising to reach a plateau where an equilibrium is established between free volume creation through STZ activation and free volume annihilation by diffusive rearrangements. The sudden free volume accumulation coincides with the onset of strain softening and highlights an increasing STZ activation rate.

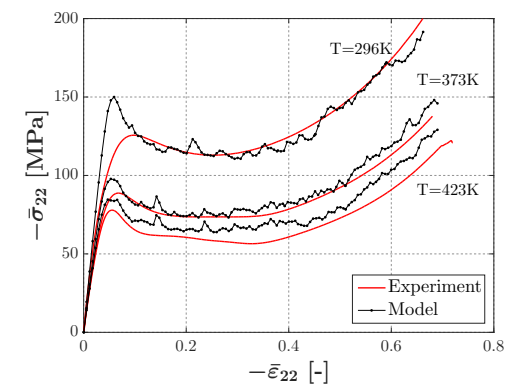

(a)

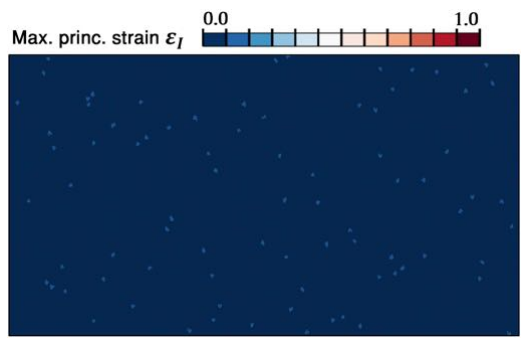

(d)

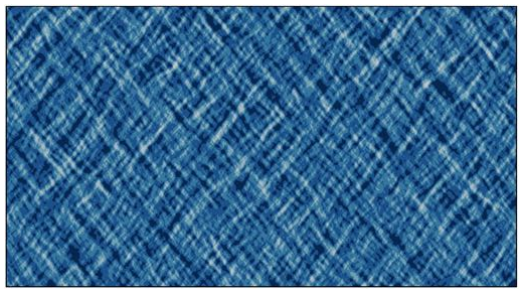

$(\mathrm{g})$

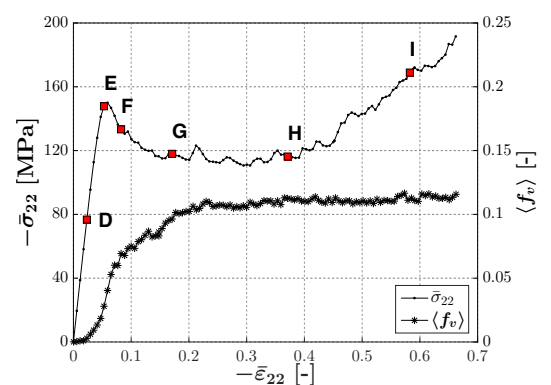

(b)

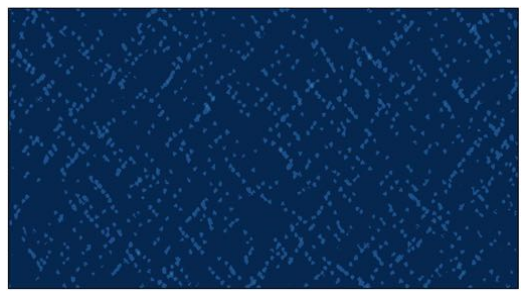

(e)

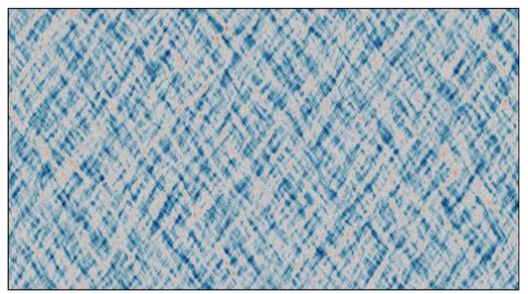

(h)

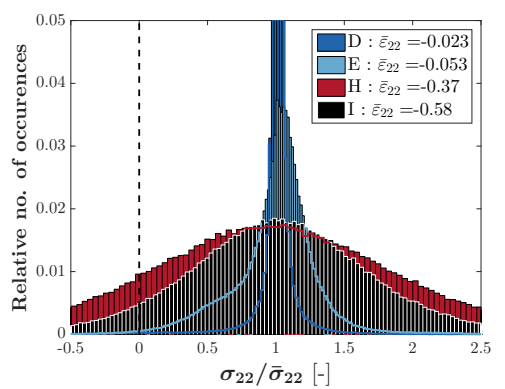

(c)

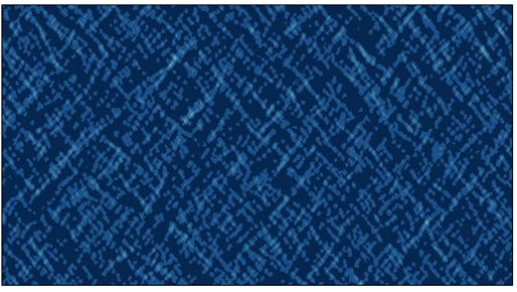

(f)

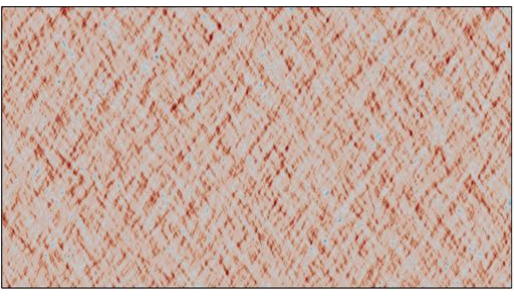

(i)

Figure 2: (a) Comparison between representative experimental stress-strain curves at $T=296 \mathrm{~K}, 373 \mathrm{~K}$ and $423 \mathrm{~K}$ under uniaxial compression and the model predictions; (b) compares the predicted evolution of the homogenized stress and of the homogenized value of the free volume variable under compression at $T=296 \mathrm{~K}$; (c) distribution of the normalized local axial stress, $\sigma_{22} / \bar{\sigma}_{22}$ corresponding to four levels of applied strain $\bar{\varepsilon}_{22}$;. (d) to (i) are contour plots of the maximum principal strain $\varepsilon_{I}$ at different simulation times corresponding to the red squares in (b). 
Fig. $2 \mathrm{~d}$ to $2 \mathrm{i}$ show the distribution of maximum principal strains $\varepsilon_{I}$ at the integration points of the FE model, corresponding to various loading stages (indicated by the red squares in Fig. 2b) during a plane strain compression test at rate $\dot{\bar{\varepsilon}}=0.01 \mathrm{~s}^{-1}$ and $T=296 \mathrm{~K}$. Prior to the onset of yielding (Fig. 2d), the homogenized response is linear elastic even though some STZs have already been activated, as evidenced by the heterogeneous strain pattern. At these early stages of loading, the perturbation of the local stress field within an activated STZ prevents a subsequent activation. However, the localized stresses are progressively erased by the increasing number of neighboring which are being activated, making the STZs once again susceptible to additional activation. The effective yield point is attained when the randomly scattered clusters of STZs reach a critical size and start propagating into micro- shear bands that can interact (Fig. 2e). At this stage, the stress and strain fields become polarized in bands oriented in the maximum shear stress direction, leading to strain localization and to softening (Fig. $2 \mathrm{f}$ and $2 \mathrm{~g}$ ). At larger applied strain, the rate of activation within the shear bands progressively decreases as the hardening contribution starts to dominate, significantly reducing further STZ activations, and hence building stress again in the band leading to the progressive re-hardening stage (Fig. $2 \mathrm{~h}$ and $2 \mathrm{i}$ ).

Fig. 2c describes - for the same simulation - the distribution of the normalized axial stress $\sigma_{22} / \bar{\sigma}_{22}$ at integration points, for four levels of applied deformation. Unsurprisingly, in the linear elastic stage $\left(\bar{\varepsilon}_{22}=-0.023\right.$, letter D in Fig. $2 b$ and 2c), the stress distribution is narrow, since the number of activated sites is low. However, in the post-yield softening regime $\left(\bar{\varepsilon}_{22}=-0.37\right.$, letter $\mathrm{H}$ in Fig. $2 \mathrm{~b}$ and $\left.2 \mathrm{c}\right)$, the stress distribution significantly broadens, highlighting the strong increase of activation rate associated with shear band propagation and the many different STZ activations associated with it. It is worth noting that, already at this stage, a non-negligible portion of the material is subjected to an axial stress in the opposite direction to the macroscopic loading one, as highlighted by the part of distribution on the left side of the dashed line in Fig. 2c, building strong back stresses in the material.

\subsection{Non-linear unloading behavior and Bauschinger effect}

Fig. 3a shows the predicted mechanical response upon full unloading (where the applied load $F=0$ at the end of the test), after having applied compressive strains up to $\bar{\varepsilon}_{22}=-0.2,-0.35$ and -0.5 . The model captures relatively well the non-linear unloading response, which is especially visible when unloading from $\bar{\varepsilon}_{22}=-0.5$. A comparison with the experiment is provided in Fig. S3 in SI, which shows experimental curves obtained in pure compression up to different strain levels followed by an unloading sequence. While the model captures the non-linear unloading response, it underestimates the amplitude of the strain recovered upon unloading.

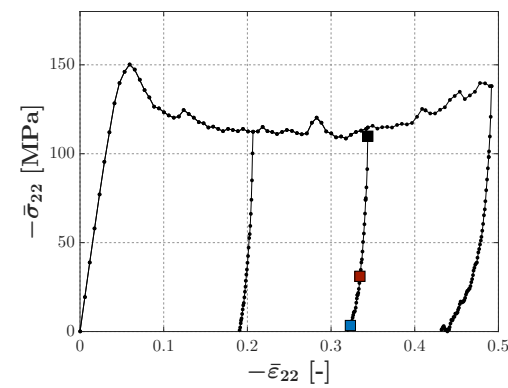

(a)

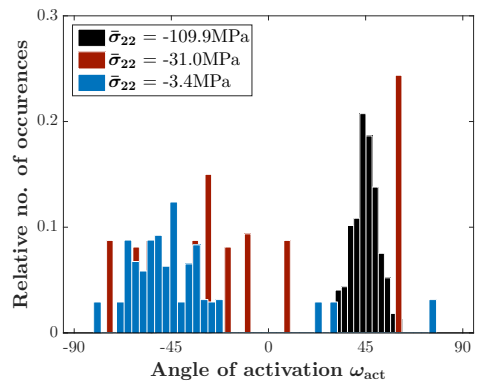

(b)

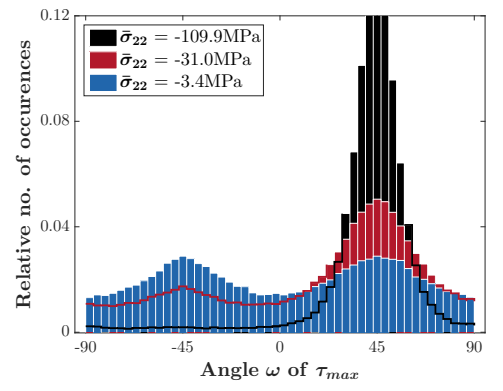

(c)

Figure 3: (a) shows the predicted unloading stress strain curves after having applied compressive strains up to $\bar{\varepsilon}_{22}=-0.2,-0.35$ and -0.5 ; (b) plots the instantaneous distribution of the STZ angles of activation $\omega_{\text {act }}$ at three stages of unloading (highlighted by the colored squares on (a)) while (c) plots the distribution of the angle $\omega$ corresponding to $\tau_{\max }$ at the same unloading stages following the compression up to $\bar{\varepsilon}_{22}=-0.35$.

In order to unravel in more details the dynamics of the STZ deformation process, Fig. 3b shows the instantaneous distribution of STZ angles of activation $\omega_{\text {act }}$ at the three different stages of unloading at $\bar{\varepsilon}_{22}=-0.35$ (illustrated by the colored squares in Fig. 3a), whereas Fig. 3c gives the distribution of $\omega$, the orientation of $\tau_{\max }$, at the same stages. At the beginning of unloading $\left(\bar{\sigma}_{22}=-109.0[\mathrm{MPa}]\right.$ in black in Fig. 3b and 3c), the local stress field is still very much polarized in the compression direction, and thus still favors forward activation. As a result, the distribution of activation angles is narrow and centered around $45^{\circ}$, the maximum shear angle under pure compression. At the intermediate unloading stage (in red in Fig. 3b and 3c), the distribution of $\omega$ starts shifting towards a bimodal 


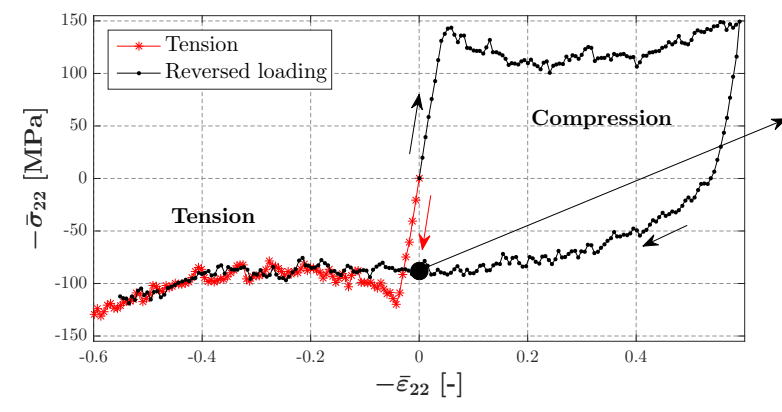

(a)

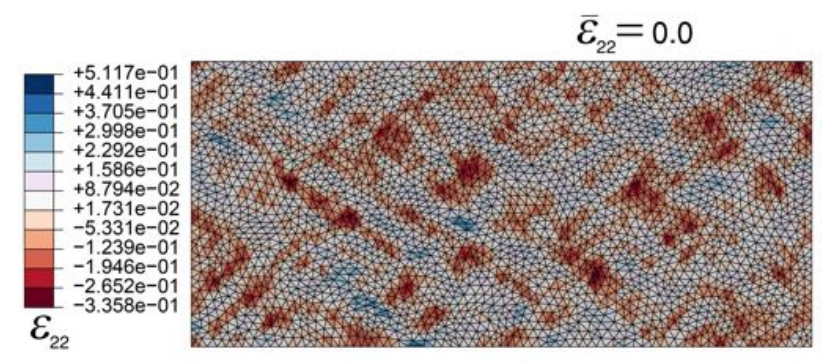

(b)

Figure 4: (a) the black dots curve shows the model response after a fully reversed loading from compression up to $\bar{\varepsilon}_{22}=-0.6$ to tension down to $\bar{\varepsilon}_{22}=0.55$, while the red stars curve corresponds to a monotonic tensile loading; (b) illustrates the highly heterogeneous distribution of $\varepsilon_{22}$ after the first compression stage of the fully-reversed loading, for $\bar{\varepsilon}_{22}=0$.

distribution, with a second peak of distribution emerging around $-45^{\circ}$. As a consequence, the instantaneous angle of activation distribution also widens. Indeed, during unloading, the magnitude of the local stress $\sigma_{22}$ decreases, concurrently decreasing the number of STZ activations in the forward direction. At the same time, a non-negligible portion of the material is already subjected to a stress of the opposite sign due to strong back stresses in the material (even prior to the beginning of unloading, as shown in Fig. 2c). As the load is reversed, these zones quickly become susceptible to backward activation, initiating the non-linearity in the unloading response. At the final loading stage (in blue in Fig. $3 \mathrm{~b}$ and $3 \mathrm{c}$ ), the distribution of angles of activation is centered around $-45^{\circ}$, which is the angle of activation in tension.

Fig. 4a shows the model response for a fully-reversed cyclic loading: the sample is subjected to compression up to $\bar{\varepsilon}_{22}=-0.6$ and is then loaded in tension up to $\bar{\varepsilon}_{22}=0.55$. The model predicts a strong Bauschinger effect with a yield stress in tension much lower than in compression. The key feature for explaining the strong Bauschinger effect upon unloading and reversed compression-tension loading lies in the explicit modeling of discrete plasticity events with specific directionality, and associated induced stress and strain field heterogeneity, as evidenced by the blue data in Fig. $3 \mathrm{~b}$ and $3 \mathrm{c}$. Moreover, the tensile part of the curve (i.e. for $\bar{\varepsilon}_{22}>0$ ) essentially recovers the tensile large strain behavior of the pristine material, in red in Fig. 4a. A notable difference is the absence of upper yield and intrinsic strain softening in the tensile part of the fully reversed loading. This effect can be explained by a typical mechanically-induced rejuvenation effect, correctly accounted for by the model. Indeed, even though the applied strain $\bar{\varepsilon}_{22}$ is brought back to zero after the first compression step (black circle in Fig. 4a), the local stress and strain distributions are still very heterogeneous in the material, as illustrated by the contour plot of $\varepsilon_{22}$ in Fig. $4 \mathrm{~b}$, in parallel with a non-zero homogenized free-volume content $\left\langle f_{v}\right\rangle>0$.

Considering this impact of the stress and strain field heterogeneity on the amplitude of the stress drop prior to strain softening provides an explanation for the overestimation of the yield stress previously mentioned when comparing the experimental results and the model response under axial compression at $T=296 \mathrm{~K}$ in Fig. 2a. The initial state of of the model is unrealistically considered as homogeneous, like if it had been aged during an infinitely long time. The stress drop would be significantly decreased by accounting for the initially heterogeneous nature of the material, coming from the non-homogeneous experimental curing process. The effect is less important at higher temperatures, as the thermal activations become more prevalent, favoring more randomly distributed activations to reaching the softening regime.

\subsection{Creep and rate-reversal phenomena}

RTM6 displays a complex behavior upon partial unloading, including forward and backward creep and ratereversal phenomenon depending on the level of the applied load (Fig. 5a). In these experiments, uniaxial compression at $T=296 K$ was applied up to $\bar{\varepsilon}_{22}=-0.25$ (associated with reaching an applied force $F_{\max }$ ), followed by a partial unloading and a creep test at a predefined value of the force $F \leq F_{\max }$. The corresponding evolution of the creep strain $\varepsilon_{\text {creep }}(t)$ (defined as the difference between the current total strain and the strain at the start of the creep test) at various levels of the applied force $F$ is illustrated in Fig. 5b. At large values of the constant applied force $\left(F \geq 0.8 F_{\max }\right)$, 
the RTM6 behavior is dominated by forward creep, with $\varepsilon_{\text {creep }}(t)>0$. At small values of the constant applied force $\left(F \leq 0.25 F_{\max }\right)$, the behavior is dominated by backward creep with $\varepsilon_{\text {creep }}(t)<0$. At intermediate values of the applied force, the behavior shows a smooth transition from backward to forward flow, called rate-reversal phenomenon Khan (2006).

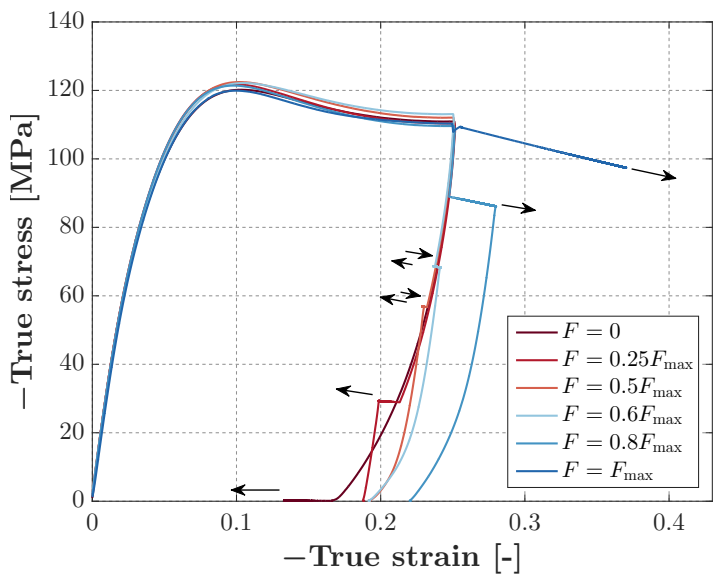

(a)

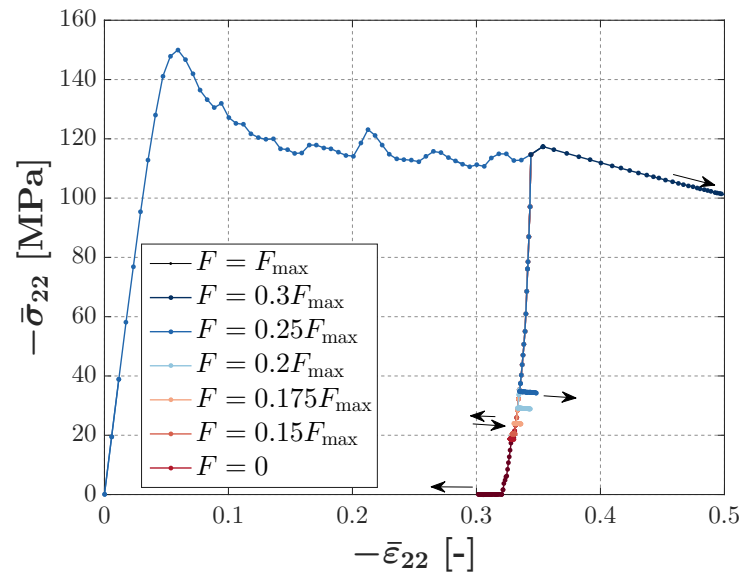

(c)

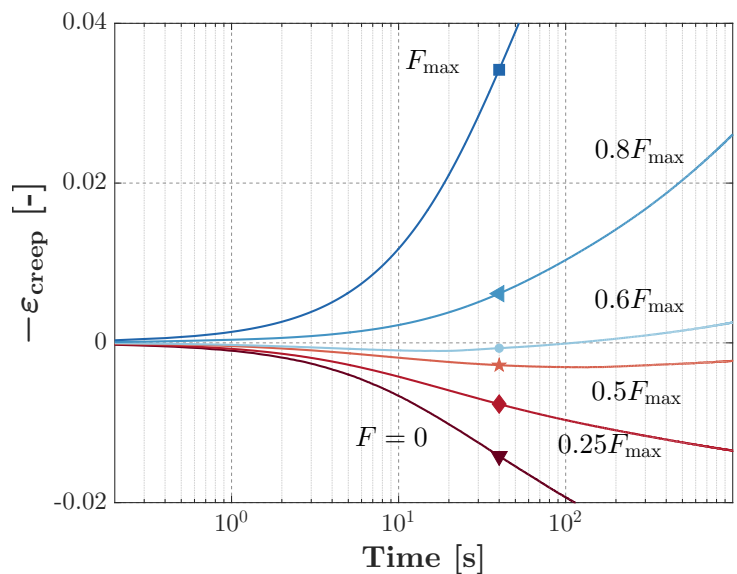

(b)

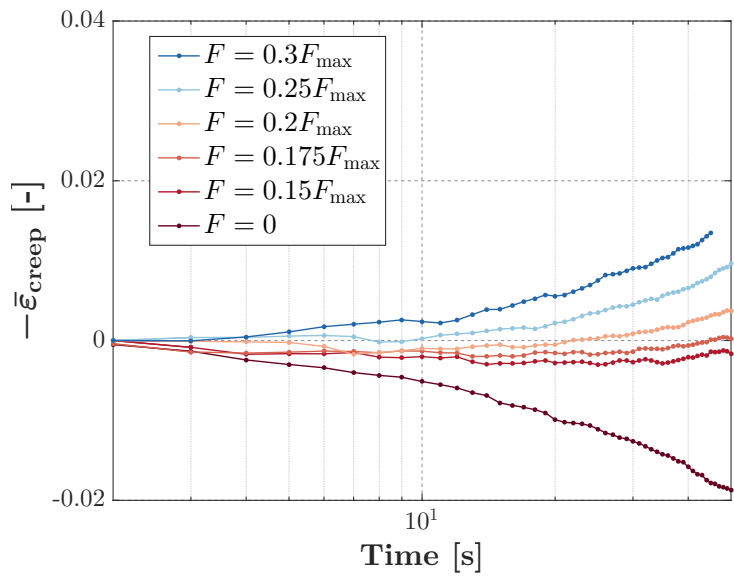

(d)

Figure 5: (a) shows the experimental stress-strain response and (b) the evolution of creep strain with time; (c) and (d) illustrate the model predictions for the creep tests, (c) showing the stress-strain response of the model and (d) the evolution of creep strain, similarly as in (b).

The predictions of the STZ model are displayed in Fig. 5c and 5d. The STZ model captures well the overall time-dependent flow behavior of RTM6, predicting forward creep when the sample is kept under high applied load and backward creep when the applied load is sufficiently low. While the predicted range of forces at which the rate-reversal phenomenon occurs does not perfectly match the experimental case, the model is able to capture this phenomenon, as shown in Fig. 5d. The effect is particularly pronounced in the model at loads between 15 and $25 \%$ of $F_{\max }$.

The rate of both forward creep and backward creep decreases with time. Fig. 6a illustrates the decrease of the instantaneous number of activations $N_{\text {act }}$ with time during a creep test at $F=F_{\max }$. The rate of STZ activation in the forward direction progressively plummets as the finite network stretchability function reduces the rate of activation of STZs despite the fact that their stress state still promotes activation in the forward loading direction, as evidenced by the mean angle of activation $\left\langle\omega_{\text {act }}\right\rangle$ that remains close to $45^{\circ}$. In the case of creep at $F=0$, the back stresses, generated by the heterogeneous nature of plastic deformation and that are responsible for the backward flow, progressively vanish as the local stress field self-equilibrates with no more external force to favor one direction over another. This 


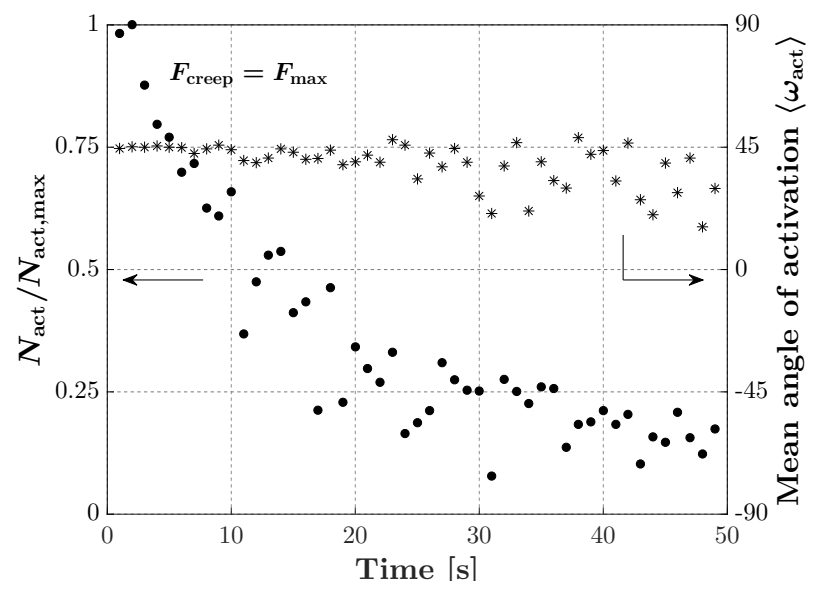

(a)

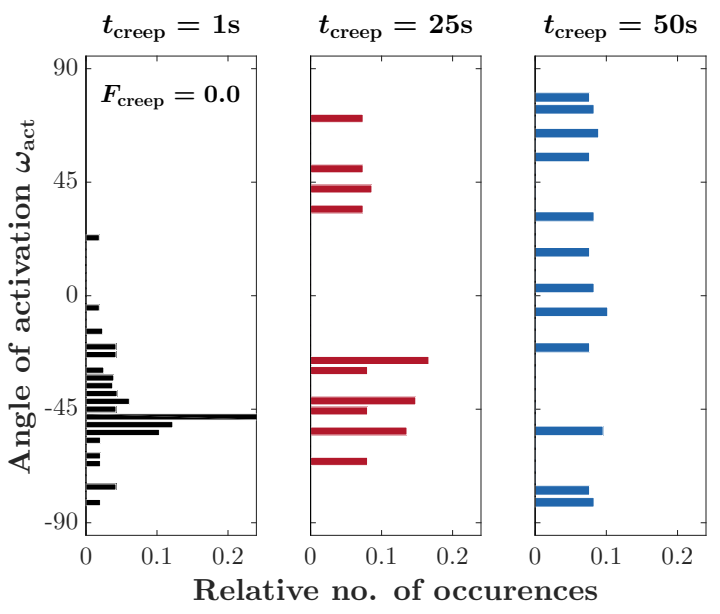

(b)

Figure 6: (a) shows in parallel, for a creep test at $F=F_{\max }$, the normalized evolution of the instantaneous number of STZ activations $N_{\text {act }} / N_{\text {act,max }}$ ( $N_{\text {act,max }}$ being the maximum value of $N_{\text {act }}$ encountered during the creep test) and the evolution of the mean angle of activation $\left\langle\omega_{\text {mathrmact }}\right\rangle$; (b) shows histograms of the instantaneous STZ angles of activation at three stages of a creep test at $F=0$.

is illustrated in Fig. 6b where the transition from a distribution of instantaneous STZ activation orientation centered around $-45^{\circ}$ in the first stages of the creep test evolves toward a fully balanced distribution at the end of the test. While a non-zero probability of further forward or backward plastic event always remains, the probability of a net flow in one direction strongly decreases with time once the external force vanishes. This is in agreement with experimental observations for RTM6, as an infinitely long time would be required to entirely recover the applied strain.

The competition between backward and forward flow responsible for the rate-reversal phenomenon in creep experiments can now be addressed through their respective activation kinetics. As already shown in Fig. $3 \mathrm{~b}$ and $6 \mathrm{~b}$, there is a direct relationship between the backward or forward nature of a strain increment and the average angle of activation $\left\langle\omega_{\text {act }}\right\rangle$ during the corresponding time increment. The correlation is such that positive creep strain increments (i.e. backward direction) are directly linked to an average angle of activation in the tensile direction (i.e. $0<\omega_{\text {act }} \leq-45^{\circ}$ ), and vice versa (see Fig. S4 in SI). Hence, the associated mechanism for rate-reversal is the occurrence of activations in the backward direction, prior to the subsequent activations in the forward direction. The backward activations are due to the back stresses generated during the loading and partial unloading phases. These tend to dominate over forward activations in the first instants of creep, as most of the back stresses have just been created, the driving force is important due to the short scale freshly perturbated local stress field while forward activations come from a more diffused effect of the overall compressive stress level operating over the entire sample. The amount of backward creep obtained prior to forward creep is thus proportional to the amplitude of the back stresses and the number of zones subjected to a stress $\sigma_{22}$ in the tensile direction.

\section{Characteristic length scale and link with microscopic observations}

The ability of the STZ model to predict the mechanical behavior of RTM6 provides a physical insight on the role of elementary plastic events on the plastic behavior of glassy polymers. However, a direct experimental evidence of the aforementioned mechanisms remains elusive. While micro and macro-clusters of shear bands have already been observed for bulk flat specimens (Bowden and Jukes, 1968; Kramer, 1974) and at the tip of notches (Ishikawa et al., 1981; Narisawa et al., 1982), the scale of a few STZs or even a single shear band has never been explored outside in-silico MD simulations. This section will not provide a definitive experimental proof of the physical existence of STZ in RTM6 but shows experimental results obtained at the micro- and nano-scale level which highlight the presence of heterogeneous deformation patterns at the scale considered by the model.

Fig. 7a, $7 \mathrm{~b}$ and $7 \mathrm{c}$ show electron micrographs at three different levels of magnification from a compression in-situ test on a unidirectional (UD) carbon fiber reinforced composite made with RTM6 as matrix. The specimens have been 


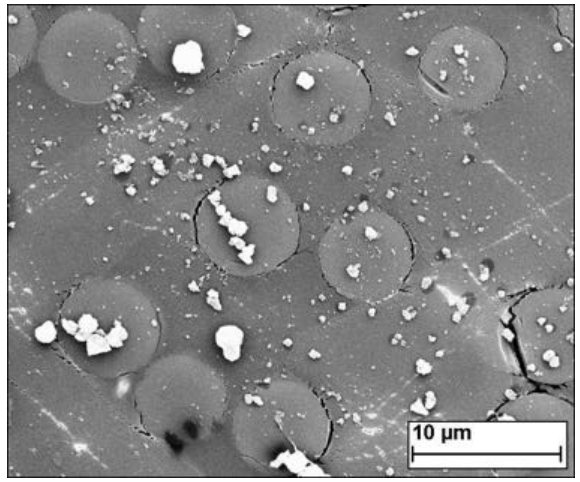

(a)

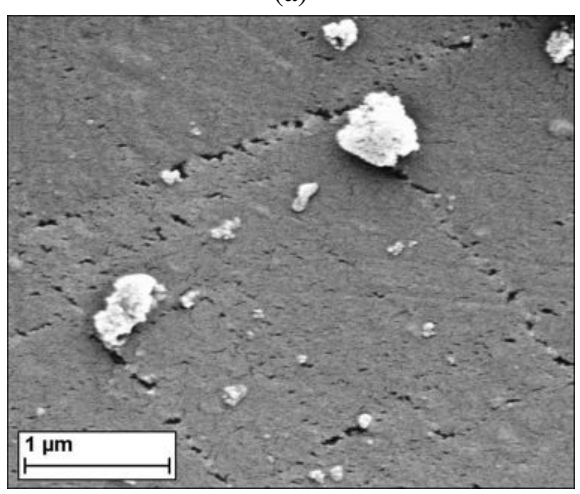

(c)

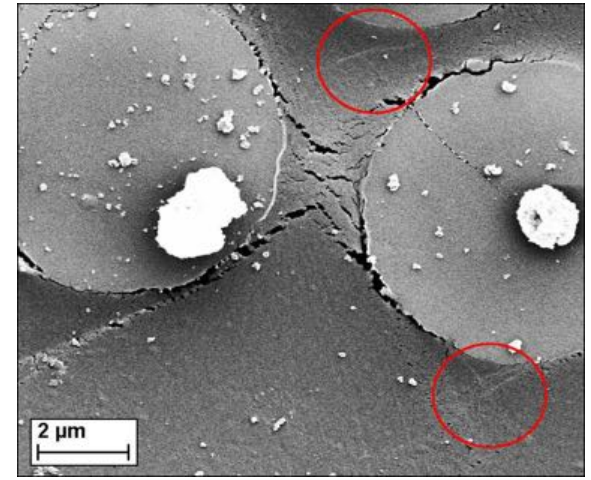

(b)

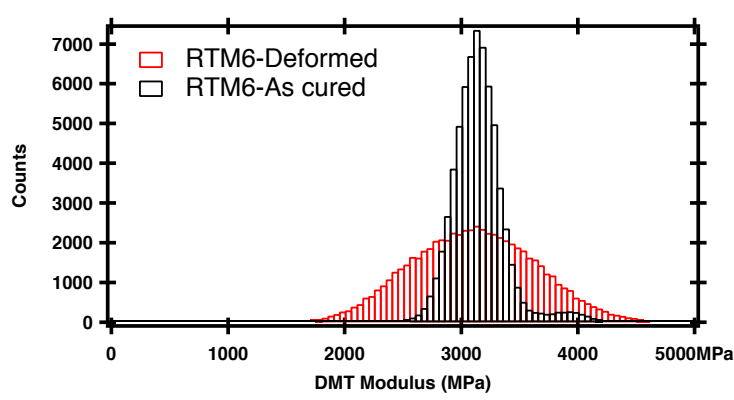

(d)

Figure 7: (a), (b) and (c) are scanning electron micrographs showing strain localization under the form of shear bands during the compression of a UD composite with RTM6 as a matrix at three different levels of magnification; d) shows the DMT modulus distribution obtained by AFM over a $0.5 \times 0.5 \mu \mathrm{m}^{2}$ zone on the surface of an undeformed specimen and a specimen compressed up to $\bar{\varepsilon}=-0.5$; 
coated by a thin gold layer prior to analysis. The three micrographs show strain localization in the matrix under the form of thin shear bands. The exact width of the shear bands is difficult to estimate but is certainly below $100 \mathrm{~nm}$. The small cracks at the specimen surface, particularly visible in Fig. 7b and 7c, are the result of local failure induced by the large shear strain in the localization bands and their opening has then led to a broadening with respect to the initial shear bands thickness. Examples of heavily sheared matrix can be seen in the top and the bottom right of Fig. $7 \mathrm{~b}$, highlighted by the red circles, where pixel wide shear bands are observed.

Fig. 7d shows Atomic Force Microscopy (AFM) histograms of the Dejarguin-Muller-Toropov (DMT) modulus Derjaguin et al. (1975) measured over a $0.5 \mu \mathrm{m}^{2}$ zone, obtained on a specimen of RTM6 before and after deforming the specimen up to $\bar{\varepsilon}=-0.5$ in uniaxial compression. As described in Bahrami et al. (2015), even in an undeformed epoxy resin, whose curing degree is over 95\%, AFM mapping reveals a heterogeneity in terms of modulus distribution, as shown in black in Fig. 7d. The width and shape of the distribution confirms that even before large deformation the mechanical landscape already includes heterogeneities. Considering the relative smoothness of the distribution, these heterogeneities must be significantly smaller than the side of the mapped surface (500nm). More importantly, Fig. 7d also shows that the DMT modulus distribution significantly broadens after deforming the specimen up to $\bar{\varepsilon}=-0.5$. The observed increase in heterogeneities within RTM6 after deformation is thus in good agreement with the STZ model predictions of Fig. 2c, confirming our working hypothesis that deformation micro-mechanisms in the glassy state are strongly heterogeneous and occur at a length scale well below $100 \mathrm{~nm}$.

\section{Conclusion}

A model has been developed to describe and predict the viscoplastic behavior of epoxy resins, based on the framework of STZ dynamics. The identification and validation of the model was performed based on a vast experimental campaign performed on the RTM6 epoxy resin. By explicitly describing the discrete elementary plastic events, the highly heterogeneous nature of plastic flow in glassy polymers at the mesoscale can be captured, including the micro shear band nucleation and propagation. The model relies on a Eyring-type equation for the rate of activation of the STZs, with a microstructural and thermo-mechanical dependent activation energy. The main findings of this work can be summarized as follows:

- The yield stress is reached when STZ clusters reach a critical size and start interacting.

- The post-yield softening is the consequence of the propagation of these STZ clusters in the form of micro shear bands.

- Rehardening is attributed to the increasing difficulty to activate a STZ multiple times in the same direction, accounting for the finite stretchability of a highly cross-linked polymer network.

- The temperature dependence of the activation volume is sufficient to capture the temperature dependent mechanical response of RTM6.

- The non-linearity upon unloading and associated Bauschinger effect can be explained by the early STZ activations in the opposite direction occurring at the early stages of unloading, due to the strong back stresses built in the material during the loading phase.

- Forward and backward creep, and the rate-reversal phenomenon, are the result of the back stress-generated competition between backward and forward activations kinetics of STZs.

The proposed approach offers an alternative to complex phenomenological constitutive models, reducing the number of parameters from more than twenty to seven, while bridging the gap between atomistic and continuum mechanics approaches by providing the missing ingredients for seamless scale transition. Moreover, its mesoscale character enables the modelling of the behavior of locally confined polymer under heterogeneous stress fields, such as bulk polymer subjected to (nano-)indentation, or an epoxy matrix confined between carbon fibers in a composite material, where homogenization-based approaches are not applicable. In this regard, efforts are currently devoted to an extension of the model to 3D. Finally, the proposed model can be used to identify the numerous parameters of phenomenological constitutive models, which remain unavoidable for the simulation of large components. 


\section{Acknowledgements}

The financial support of the Fonds de la Recherche Scientifique de Belgique (FNRS) is acknowledged through the FNRS doctoral fellowship of XPM and the FRIA doctoral fellowship of JC. XPM also acknowledges the financial support of the Belgian American Educational Foundation (Cabeaux-Jacobs 2015). The authors would like to thank P.P. Camanho for fruitful discussions during the course of this work. Computational resources have been provided by the supercomputing facilities of the Université catholique de Louvain (CISM/UCL) and the Consortium des Equipements de Calcul Intensif en Fédération Wallonie Bruxelles (CECI) funded by the Fonds de la Recherche Scientifique de Belgique (FRS-FNRS).

\section{References}

Ames, N. M., Srivastava, V., Chester, S. a., Anand, L., aug 2009. A thermo-mechanically coupled theory for large deformations of amorphous polymers. Part II: Applications. International Journal of Plasticity 25 (8), 1495-1539.

Anand, L., Ames, N. M., Srivastava, V., Chester, S. a., 2009. A thermo-mechanically coupled theory for large deformations of amorphous polymers. Part I: Formulation. International Journal of Plasticity 25 (8), 1474-1494.

Anand, L., Gurtin, M. E., 2003. A theory of amorphous solids undergoing large deformations , with application to polymeric glasses. International Journal of Solids and Structures 40, 1465-1487.

Argon, A., Bessonov, M., 1977. Plastic deformation in polyimides, with new implications on the theory of plastic deformation of glassy polymers. Philosophical Magazine 35 (4), 917-933.

Argon, A., Bulatov, V., Mott, P., Suter, U., 1995. Plastic deformation in glassy polymers by atomistic and mesoscopic simulations. Journal of Rheology 39 (2), 377-399.

Argon, A. S., 1973. A theory for the low-temperature plastic deformation of glassy polymers. Philosophical Magazine 28 (4), $839-865$.

Argon, A. S., 1979. Plastic Deformation in Metallic Glasses. Acta Metallurgica 27, 47-58.

Argon, A. S., 2013. The Physics of Deformation and Fracture of Polymers. New York.

Bahrami, A., Morelle, X., Hng Minh, L., Pardoen, T., Bailly, C., Nysten, B., 2015. Curing dependent spatial heterogeneity of mechanical response in epoxy resins revealed by atomic force microscopy. Polymer (United Kingdom) 68, 1-10.

Bergstrom, J., Boyce, M., 2001. Constitutive modeling of the time-dependent and cyclic loading of elastomers and application to soft biological tissues. Mechanics of Materials 33 (9), 523-530.

Bowden, P., Jukes, J., 1968. The plastic yield behaviour of polymethylmethacrylate. Journal of Materials Science 3 (2), 183-190.

Bowden, P., Raha, S., 1974. A molecular model for yield and flow in amorphous glassy polymers making use of a dislocation analogue. Philosophical Magazine 29 (1), 149-166.

Boyce, M. C., Parks, D. M., Argon, A. S., 1988. Large inelastic deformation of glassy polymers. part I: rate dependent constitutive model. Mechanics of Materials 7 (1), 15-33.

Budrikis, Z., Castellanos, D. F., Sandfeld, S., Zaiser, M., Zapperi, S., 2017. Universal features of amorphous plasticity. Nature Communications 8 (May), 1-10.

Bulatov, V. V., Argon, A. S., 1994. A stochastic model for continuum elasto-plastic behavior : I . Numerical approach and strain localization. Modelling Simulations in Materials Science Engineering 2, 167-184.

Chen, K., Schweizer, K. S., may 2011. Theory of Yielding, Strain Softening, and Steady Plastic Flow in Polymer Glasses under Constant Strain Rate Deformation. Macromolecules 44 (10), 3988-4000.

Dawnkaski, E., Srivastava, D., Garrison, B., 1995. Time dependent monte carlo simulations of h reactions on the diamond 001(21) surface under chemical vapor deposition conditions. The Journal of Chemical Physics 102 (23), 9401-9411.

Derjaguin, B., Muller, V., Toporov, Y., 1975. Effect of contact deformations on the adhesion of particles. Journal of Colloid And Interface Science 53 (2), 314-326.

Ediger, M. D., 2000. Spatially Heterogeneous Dynamics in Supercooled Liquids. Annual Review in Physical Chemistry 51, 99-128.

Eshelby, J., 1957. The determination of the elastic field of an ellipsoidal inclusion, and related problems. Proceedings of the Royal Society Part A 241 (1226), 376-396.

Eyring, H., 1936. Viscosity, plasticity, and diffusion as examples of absolute reaction rates. The Journal of Chemical Physics 4 (4), $283-291$.

Falk, M., Langer, J., Pechenik, L., 2004. Thermal effects in the shear-transformation-zone theory of amorphous plasticity: Comparisons to metallic glass data. Physical Review E - Statistical, Nonlinear, and Soft Matter Physics 70 (1 1), 011507-1-011507-11.

Falk, M. L., Langer, J. S., 1998. Dynamics of Viscoplastic Deformation in Amorphous Solids. Physical Review E - Statistical, Nonlinear, and Soft Matter Physics 57 (6), 16.

Fiedler, B., Hojo, M., Ochiai, S., Schulte, K., Ando, M., 2001. Failure behavior of an epoxy matrix under different kinds of static loading. Composites Science and Technology 61 (11), 1615-1624.

Gerlach, R., Siviour, C., Petrinic, N., Wiegand, J., 2008. Experimental characterisation and constitutive modelling of rtm-6 resin under impact loading. Polymer 49 (11), 2728-2737.

G'Sell, C., 1986. Plastic Deformation of Glassy Polymers : Constitutive Equations and Macromolecular Mechanisms. In: 7th International Conference on the Strength of Metals and Alloys (ICSMA 7). Montreal, Canada, pp. 1943-1982.

Gudimetla, M., Doghri, I., 2017. A finite strain thermodynamically-based constitutive framework coupling viscoelasticity and viscoplasticity with application to glassy polymers. International Journal of Plasticity 98, 197-216.

Harris, M. B., Watts, L. S., Homer, E. R., 2016. Competition between shear band nucleation and propagation across rate-dependent flow transitions in a model metallic glass. Acta Materialia 111, 273-282. 
Hasan, O. A., Boyce, M. C., 1995. A Constitutive Model for the Nonlinear Viscoelastic Viscoplastic Behavior of Glassy Polymers. Polymer Engineering \& Science 35 (4), 331.

Hasan, O. A., Boyce, M. C., Li, X. S., Berko, S., feb 1993. An investigation of the yield and postyield behavior and corresponding structure of poly(methyl methacrylate). Journal of Polymer Science Part B: Polymer Physics 31 (2), 185-197.

Haward, R., Thackray, G., 1968. The use of a mathematical model to describe isothermal stress-strain curves in glassy thermoplastics. Proceedings of the Royal Society of London A: Mathematical, Physical and Engineering Sciences 302 (1471), 453-472.

Homer, E. R., Schuh, C. A., 2009. Mesoscale modeling of amorphous metals by shear transformation zone dynamics. Acta Materialia 57 (9), 2823-2833.

Hoy, R., Robbins, M., 2006. Strain hardening of polymer glasses: Effect of entanglement density, temperature, and rate. Journal of Polymer Science, Part B: Polymer Physics 44 (24), 3487-3500.

Hoy, R., Robbins, M., 2007. Strain hardening in polymer glasses: Limitations of network models. Physical Review Letters 99 (11).

Hoy, R. S., Robbins, M. O., 2008. Strain hardening of polymer glasses: Entanglements, energetics, and plasticity. Physical Review E - Statistical, Nonlinear, and Soft Matter Physics 77 (3), 1-14.

Ishikawa, M., Ogawa, H., Narisawa, I., 1981. Brittle fracture in glassy polymers. Journal of Macromolecular Science, Part B 19 (3), $421-443$.

Jatin, Sudarkodi, V., Basu, S., 2013. Investigations into the origins of plastic flow and strain hardening in amorphous glassy polymers. International Journal of Plasticity 56, 139-155.

Khan, F., 2006. Loading history effects on the creep and relaxation behavior of thermoplastics. Journal of Engineering Materials and Technology, Transactions of the ASME 128 (4), 564-571.

Kramer, E., 1974. The stress-strain curve of shear-banding polystyrene. Journal of Macromolecular Science, Part B 10 (1), 191-202.

Langer, J., 2008. Shear-transformation-zone theory of plastic deformation near the glass transition. Physical Review E - Statistical, Nonlinear, and Soft Matter Physics 77 (2).

Li, L., Homer, E. R., Schuh, C. A., 2013. Shear transformation zone dynamics model for metallic glasses incorporating free volume as a state variable. Acta Materialia 61 (9), 3347-3359.

Malekmotiei, L., Samadi-Dooki, A., Voyiadjis, G. Z., 2015. Nanoindentation Study of Yielding and Plasticity of Poly(methyl methacrylate). Macromolecules 48 (15), 5348-5357.

Mangion, M. B. M., Cavaillé, J. Y., Perez, J., 1992. Plastic Mechanical Response of Amorphous Polymers. Philosophical Magazine A 66 (5), 773-796.

Masurel, R., Gelineau, P., Cantournet, S., Dequidt, A., Long, D., Lequeux, F., Montes, H., 2017. Role of dynamical heterogeneities on the mechanical response of confined polymer. Physical Review Letters 118 (4).

Masurel, R. J., Cantournet, S., Dequidt, A., Long, D. R., Montes, H., Lequeux, F., 2015. Role of Dynamical Heterogeneities on the Viscoelastic Spectrum of Polymers: A Stochastic Continuum Mechanics Model. Macromolecules 48 (18), 6690-6702.

Meijer, H., Govaert, L., 2005. Mechanical performance of polymer systems: The relation between structure and properties. Progress in Polymer Science (Oxford) 30 (8-9), 915-938.

Morelle, X., 2015. Mechanical characterization and physics-based modeling of a highly-crosslinked epoxy resin. PhD dissertation, Université catholique de Louvain.

Morelle, X., Chevalier, J., Bailly, C., Pardoen, T., Lani, F., 2017. Mechanical characterization and modeling of the deformation and failure of the highly crosslinked rtm6 epoxy resin. Mechanics of Time-Dependent Materials 21 (3), 419-454.

Mott, P., Argon, A., Suter, U., 1993. Atomistic modelling of plastic deformation of glassy polymers. Philosophical Magazine A: Physics of Condensed Matter, Structure, Defects and Mechanical Properties 67 (4), 931-978.

Narisawa, I., Murayama, T., Ogawa, H., 1982. Internal fracture of notched epoxy resins. Polymer 23 (2), 291-294.

Nguyen, V.-D., Lani, F., Pardoen, T., Morelle, X., Noels, L., 2016. A large strain hyperelastic viscoelastic-viscoplastic-damage constitutive model based on a multi-mechanism non-local damage continuum for amorphous glassy polymers. International Journal of Solids and Structures 96, 192-216.

Oleynik, E., 1989. Plastic deformation and mobility in glassy polymers. Progress in Colloid \& Polymer Science 80, 140-150.

Poulain, X., Benzerga, A., Goldberg, R., 2014. Finite-strain elasto-viscoplastic behavior of an epoxy resin: Experiments and modeling in the glassy regime. International Journal of Plasticity 62, 138-161.

Poulain, X., Kohlman, L., Binienda, W., Roberts, G., Goldberg, R., Benzerga, A., 2013. Determination of the intrinsic behavior of polymers using digital image correlation combined with video-monitored testing. International Journal of Solids and Structures 50 (11-12), 1869-1878.

Ree, T., Eyring, H., 1955. Theory of non-Newtonian flow. I. Solid plastic system. Journal of Applied Physics 26 (7), 793-800.

Robertson, R. E., 1966. Theory for the plasticity of glassy polymers. The Journal of Chemical Physics 44 (10), 3950-3956.

Rottler, J., 2009. Fracture in glassy polymers : a molecular modelling perspective. Journal of Polymer Science Part B: Polymer Physics, 1-19.

Samadi-Dooki, A., Malekmotiei, L., Voyiadjis, G., 2016. Characterizing shear transformation zones in polycarbonate using nanoindentation. Polymer (United Kingdom) 82, 238-245.

Senden, D., Van Dommelen, J., Govaert, L., 2010. Strain hardening and its relation to bauschinger effects in oriented polymers. Journal of Polymer Science, Part B: Polymer Physics 48 (13), 1483-1494.

Shi, Y., Falk, M., 2005. Strain localization and percolation of stable structure in amorphous solids. Physical Review Letters 95 (9).

Srivastava, V., Chester, S. a., Ames, N. M., Anand, L., aug 2010. A thermo-mechanically-coupled large-deformation theory for amorphous polymers in a temperature range which spans their glass transition. International Journal of Plasticity 26 (8), 1138-1182.

Stachurski, Z., 1997. Deformation mechanisms and yield strength in amorphous polymers. Progress in Polymer Science (Oxford) 22 (3), $407-474$.

Sundararaghavan, V., Kumar, a., 2013. Molecular dynamics simulations of compressive yielding in cross-linked epoxies in the context of Argon theory. International Journal of Plasticity 47, 111-125.

Tervoort, T., Klompen, E. J., Govaert, L. E., 1996. A multi-mode approach to finite, three-dimensional, nonlinear viscoelastic behavior of polymer glasses. Journal of Rheology 40 (5), 779.

Van Melick, H., Govaert, L., Meijer, H., 2003. On the origin of strain hardening in glassy polymers. Polymer 44 (8), $2493-2502$.

Voyiadjis, G., Malekmotiei, L., Samadi-Dooki, A., 2018. Indentation size effect in amorphous polymers based on shear transformation mediated 
plasticity. Polymer (United Kingdom) 137, 72-81.

Voyiadjis, G. Z., Samadi-Dooki, A., 2016. Constitutive modeling of large inelastic deformation of amorphous polymers: Free volume and shear transformation zone dynamics. Journal of Applied Physics 119 (22).

Wang, S.-q., Cheng, S., Lin, P., Li, X., 2014. A phenomenological molecular model for yielding and brittle-ductile transition of polymer glasses. The Journal of Chemical Physics 141, 1-19.

Wang, S.-Q., Wang, Y., Cheng, S., Li, X., Zhu, X., Sun, H., 2013. New experiments for improved theoretical description of nonlinear rheology of entangled polymers. Macromolecules 46 (8), 3147-3159.

Wu, P., Van Der Giessen, E., 1993. On improved network models for rubber elasticity and their applications to orientation hardening in glassy polymers. Journal of the Mechanics and Physics of Solids 41 (3), 427-456. 